\section{CARDIAC RESUSCITATION}

BY

W. B. PRIMRose, M.B., Сн.B.

SENIOR ANAESTHETIST, GLASGOW ROYAL INFIRMARY

The risk of cardiac failure occurring under anaesthesia, although very small, is one that has to be accepted by patient, surgeon, and anaesthetist. Some hundreds of patients die upon the operating table, or rather under anaesthesia, in this country every year. That this should be so is not remarkable from the very nature of surgery and the ailments it seeks to alleviate and cure, but when sound and healthy lives are lost-often during the performance of trifling operations-means to prevent such accidents become of prime importance.

Prevailing knowledge of cardiac failure and its treatment may be gathered from a perusal of any of the numerous works upon anaesthetics. Many reasonable recommendations are given, the last usually being that if the other methods have failed to bring about recovery direct cardiac massage may be tried. Experience is gradually accumulating to show that in cases of complete cardiac failure nothing is of any use but direct cardiac massage, and that there is a definite time within which it must be carried out. If this is observed success may be expected in healthy cases, but if the time limit is allowed to expire, while successful restarting may be accomplished, the patient will not live for more than a few hours.

Meeting with an occasional case of cardiac collapse when working with Mr. G. C. Swanson on " receiving nights " in the Glasgow Royal Infirmary during the past few years, it was the practice to do everything recommended, finally opening the abdomen and carrying out cardiac massage. In one case the heart resumed action after about a quarter of an hour's active massage, even although it had been stopped for about the same length of time. It stopped twice before finally continuing, but the patient died about eight hours later without recovering consciousness. In one or two subsequent cases less time was spent upon artificial respiration, etc., and cardiac massage was commenced earlier with better results. The heart started much more easily and remained in action longer, one case living for about twenty hours. From the behaviour of another case it was ascertained that the brain could not stand arrested circulation even for six minutes, for again death took place many hours later, showing that the cerebral circulation had never resumed completely and that stagnation had slowly spread until the medullary centres succumbed.

From these unfortunate instances we judged that for complete success cardiac massage must be carried out within three minutes of the visible onset of the failure, and that from the moment of failure artificial circulation was the urgent requirement of the dying patient, artificial respiration being of secondary importance. It was also evident, from the ease with which the healthy heart could be started, that the organ was not poisoned in these cases, but had merely " stalled," its nervous control having relaxed to too great an extent. This is an important point, because a heart poisoned by any kind of toxaemia will either not respond at all or will do so only feebly and intermittently for a little while.

This information, while of the utmost value, cannot be applied to every case of collapse that occurs during anaesthesia. One requires to know definitely that the failure is complete before embarking upon an abdominal incision. For this purpose we use amyl nitrite, which, when inhaled, has such a stimulating effect upon the depressed heart. The ordinary "faint" recovers immediately with an artificial inhalation of a capsule of this drug, whereas the arrested heart cannot respond at all We thus get an immediate diagnosis of the condition within one minute. A second capsule may be tried to make sure, but we have not found failure with the first capsule to lead us astray in diagnosing a complete failure.

Failure to produce recovery with amyl nitrite will be found to coincide with the visible, palpable, and audible evidence of cardiac arrest, and is the sign for immediate opening of the upper abdomen. In many cases it is likely that both the surgeon and the abdomen are ready for operation, in which case the matter is very simple; but conditions may be quite otherwise. The anaesthetist may find that he alone possesses the knowledge that is required to save the life of the patient. He must make the best effort he can at preparation of his hands and the abdominal skin, but whatever happens the heart must be started by direct manipulation within the aforesaid three minutes.

\section{Technique of Applying Cardiac Massage}

The opening of the abdomen for cardiac massage is a very simple matter. An incision sufficiently long to admit the hand is made in the middle line a little below the xiphisternum. Confirmation of the diagnosis will be given by the lack of bleeding and the post-mortem colour of the muscles, which are extremely relaxed. The perito. neum is opened with a little care for the liver-the other viscera have usually fallen back in the general relaxation.

The right hand, inserted through the wound, passes above the liver to the left subphrenic region, where the heart may be felt at rest through the diaphragm. The left hand is placed over the praecordium, the heart thus lying between the two hands. The operator then has to imitate the cardiac beat to the extent of making the ventricular part expel blood, and, to begin with, a rapid rate of compression is adopted for about ten to fifteen seconds, to irritate the organ a little, followed by the pressure at the normal rate of beating. When the heart reacts the peculiar sensation of a living thing slipping under the hand announces successful restarting, and once started it only suffices to wait for a few minutes to be sure that the beat is maintained.

The term " massage" does not convey the correct idea of what is required to restart a heart: it suggests placid stroking of the organ. One of the failures in my own experience was due to a surgeon undertaking the resuscitation himself on these lines. When he handed over to me, after trying for a few minutes, more energetic treatment restarted the heart promptly; but the time was past, and the patient died about twelve hours later. The surgeon said he had no idea that the heart could be so vigorously compressed, nor that such vigorous treatment was necessary for effective recovery. The fear is not of damaging the heart (which is a very tough viscus) by this treatment, but of damaging the diaphragm, which is much more delicate. I am not aware of the latter having occurred in any case.

The position adopted by the hands is a very awkward one, as only the fingers of the right hand are available for compressing the heart. The operator, working at such muscular disadvantage, should not be surprised at a feeling of fatigue in his arms in a very short time, and perspiration is usual if the effort is at all prolonged. These incidentals are mentioned merely to complete the idea of the energy that may be required to restart a heart, particularly in a case in which recovery may be doubtfulfor example, in a toxic subject. In these cases massage may be prolonged for some time before being finally successful or otherwise. If possible, oxygen should be given, since the vigorous manipulations effect a certain amount of artificial respiration during the massage. It is definitely beneficial, as may be seen by the colour of the face ; and this, along with the artificial pulse that is 
usually palpable at the wrist, and the diminishing pupils, gives a remarkable imitation of vitality.

\section{Results in Four Cases}

From this point onwards we seemed to be in a better position for dealing with the emergency of cardiac failure, for, although we had been denied complete success, much had been learned for future application should occasion arise. Occasions have arisen during the last three years, and we are able to record four successful cases.

Case 1.-This occurred with a senior surgical colleague, Mr. Milne McIntyre of the Royal Infirmary, to whom I had imparted my views. The case was one of removal of an interval appendix, a resident giving the anaesthetic, mostly chloroform. Collapse set in shortly after the commencement of the operation. As there was no sign of recovery with artificial respiration and adrenaline injected into the heart muscle, Mr. McIntyie opened the abdomen higher up and felt for the heart, which was completely at rest. With very little effort at restarting it took up its beat vigorously and permanently, the patient making a complete recovery.

Case 2.-This was with Mr. J. Mill Renton of the Western Infirmary, whose own experience of cardiac resuscitation was leading him to early intervention. The operation was for removal of a small growth from the abdominal wall of a young woman. I was giving the anaesthetic, in which chloroform figured in the ethyl chloride-ether-chloroform sequence along with oxygen. The collapse was of the usual dramatic kind. Artificial respiration with amyl nitrite was tried without success ; adrenaline was also used without effect. Mr. Renton then opened the abdomen and found the heart quite still. After a little manipulation it set off vigorously and permanently, the patient recovering completely. This case was of interest, for the immediate recovery from the ordeal was slow, and the patient, after being in bed for about half an hour, showed marked cerebral irritation, two nurses being required to keep her in bed. This, however, yielded to morphine (1/4 grain), and when she awakened from the sleep so produced her behaviour was quite normal, though there was a gap in her memory for recent events, which disappeared during the next thirty-six hours. The nature of the recovery here suggested a borderline case, caught just within the time limitsay, four minutes from the onset.

Case 3.-This was more of an incident to the operation, which was gastro-enterostomy in a male subject. The surgeon was Mr. G. C. Swanson; a resident gave the anaesthetic. The collapse occurred after the abdomen had been opened, and Mr. Swanson noticed it almost from its commencement. Without preliminaries as to diagnosis, he inserted his hand through the existing incision and found the heart quite stopped. A little manipulation set it going, and the operation was continued to its conclusion, recovery being complete.

Case 4.-This occurred in my hands while I was anaesthetizing a mastoid case in a young man for Mr. James Harper of the Royal Infirmary. A C.E. mixture with oxygen was being used. Collapse occurred suddenly, about ten minutes from end of operation. Mr. Harper resolutely applied artificial respiration, of which I made use in getting a capsule of amyl nitrite inhaled and absorbed. Meanwhile, after a rapid "scrub-up" and the donning of overalls and gloves, I was ready for the now more than likely abdominal incision, for the other measures had produced nothing in the way of recovery. With hasty preparation of the skin, the abdominal wall was incised and the peritoneum opened. The heart was easily felt, soft and at rest. About half a minute's manipulation sufficed to start it, and the beat was maintained steadily and permanently. Recovery was complete in ali respects, although the incision incited some wonder on the part of the patient. In this case closing the abdomen presented a little difficulty to me, unskilled in the art. This was due to the rather rapid recovery of the patient from the anaesthetic now that his circulation was going. A nurse had to administer a little chloroform while I closed the wound, and Mr. Harper finished his operation. I was told afterwards by a surgeon that, under the circumstances; closure by means of "through and through" silkworm would have sufficed and been simpler than closing by layers.

\section{Discussion and Conclusions}

Commenting upon these cases it is evident that we are dealing with a purely mechanical breakdown, however it has been produced. The correction of the fault is also purely mechanical. That is why success is so often possible. Compare such a breakdown with, say, suppression of urine or massive collapse of a lung, conditions also occasionally associated with the giving of anaesthetics. We are quite unable to intervene with curative effect in such cases.

A word or two may be said regarding the use of adrenaline, which is at present much favoured in connexion with the treatment of cardiac failure. In these four successful cases no effect of any value was produced by the drug in the first and second cases, which received it, and it was obviously not required in the third and fourth cases, which did not get it. In another recent case of cardiac failure on the table in a young girl massage was unavailing owing to septic toxaemia. Adrenaline was then injected into the heart, after which cardiac massage was again tried, and was most effective. The heart's action, however, only lasted some five minutes, after which neither adrenaline nor massage was of any use. In another two failures belonging to this period-one an obese subject with intestinal obstruction, and the other a case of toxic exophthalmic goitre-adrenaline failed to produce any effect that could be noticed. Briefly, we would state that from our experience adrenaline is of little use in restarting a heart. It cannot reliably initiate pulsation, although it may produce ventricular spasm. Its action is also of very short duration, probably owing to the fact that adrenaline is quickly destroyed in the blood. In spite of occasicnal cases where this drug seems to play a part, reliance cannot be placed upon it at the expense of valuable time in cases of complete cardiac failure.

As already mentioned, a condition necessary for success is that the patient shall be in fair bodily health. We have had experience of failure in several cases, apart from those mentioned in connexion with the time limit, and we might sum it up by saying that if life is being threatened by any toxic condition such as acute sepsis, advanced diabetes, uraemia, thyrotoxaemia, cachexia, or old age a failure is most likely to resist all efforts at resuscitation and to remain permanent.

In view of what has been said about cardiac failure and its management, we believe that our position in regard to this special risk has considerably improved, for we feel that we should be able to save all the healthy victims and to give the others the best chance in a somewhat embarrassed fight for life.

A joint conference of the Invalid Children's Aid Association and the Central Council for the Care of Cripples will be held on November 7th and 8th at the Drapers' Hall, Throgmorton Street, London, to consider the welfare of cripples and invalid children. It will be opened by the Minister of Health, Sir Kingsley Wood. The subjects for discussion are: infectious diseases and their after-effects, with reference to immunization and other preventive measures; the problem of physical handicap in modern life, with a review of the parts played by local authorities and voluntary associations in fitting the physically handicapped person for modern life; and the after-effects of accidents, including a study of rehabilitation methods, co-operation between cottage, general, and orthopaedic hospitals in the treatment and after-care of accident cases, the problems of industrial accidents, and workmen's compensation legislation. Visits to various institutions will be arranged. The payment of delegates' expenses has been officially sanctioned. The conference fee is $7 \mathrm{~s}$. 6d., and further information may be obtained from the honorary secretary of either organization, Carnegie House, 117, Piccadilly, W.1. 